\title{
Spectral Imaging and X-Ray Microanalysis with Multiple Detectors
}

\author{
R. Wuhrer * and K. Moran ${ }^{*, * *}$ \\ ${ }^{*}$ Microstructural Analysis Unit, University of Technology, Sydney, Broadway, Australia. \\ *** Moran Scientific Pty Ltd, P.O. Box 651, Goulburn, NSW, 2580 Australia.
}

We have progressively refined our requirements for high quality x-ray mapping, which has been a process of learning, advancing our hardware and redefining our requirements. Mapping is not a simple 'one size fits all' scenario. There are still simple applications where dot mapping, especially with a wavelength dispersive spectrometer, can achieve the required results. The good thing about mapping is that there is always something new that can be learned and we are continually pushing back the boundaries of what can be achieved with mapping. It won't be far into the future that we will have an electron microscope specifically set up to do live x-ray imaging, as we now do for electron imaging. The biggest problem we face is one of being able to determine the level of sophistication in our treatment of the data collected. As with automatic peak identification and standardless analysis it is very easy to produce bad results [1]. The good news is that if you are prepared to set your system up for high quality standards analysis, then most of these problems disappear.

Most recently we have defined our general aims for high quality mapping and it should be no surprise to see that this is the same requirement for high quality EDS analysis. The general aims of high quality EDS mapping is to: 1) map at high energy resolution ( $<140 \mathrm{eV} \mathrm{Mn}), 2)$ use low beam current (preferably $<1 \mathrm{nA}), 3$ ) use large crystal area for high collection efficiency $\left.\left(>30 \mathrm{~mm}^{2}\right), 4\right)$ have low pulse pileup, 5) have no shift in resolution, gain or zero with count rate $(<1 \mathrm{eV}$ over the count rate range), 6) use a beam diameter less than the pixel resolution, 7) collect the full spectrum at each pixel with quantitation processing in the "usual" fashion and 8) perform maps at count rates greater than $30 \mathrm{kcps}$ throughput with one or more detectors. This last point is for fast imaging rather than high quality analysis.

Quantitative X-ray mapping (QXRM) using multi-detectors and combined detectors such as WDS/EDS, not only reduces mapping time, but also improves the ability to map minor and trace elements very accurately. One interesting outcome of this is that we now need to improve the collection efficiency of our WDS detectors to enable them to perform at these lower beam currents. To take advantage of this increased precision and sensitivity of QXRM, careful attention to all those parameters necessary for high quality analysis is required. Important characteristics such as resolution, gain zero calibration, pulse pileup and other artifacts need to be well characterized for each detector that is being used. The final image is then created from the fully background corrected, overlap corrected and inter-element corrected calculation at each pixel in the image. As there is a very large dynamic concentration range to be considered ( 5 orders of magnitude) it is important to understand that the precision at each level will depend on how well this characterization is carried out. The low concentration range requires much more careful work than for major elements. To achieve this, time is no longer an issue but a means to accomplish a more precise result. Thus, if we can introduce more efficient detection then we can achieve this result in a shorter time. This may be achieved by counting more x-rays with one detector, so long as the critical quantitative characteristics of the detector is not compromised, or can easily be achieved by adding more 
detectors. As a check for consistency between detectors, a technique was developed that involves assigning a different RGB colour to each detector for the same element [2]. By doing this, when we combine the three maps of the same element, we should obtain a grey scale map, not a colour map. A grey scale indicates total correlation between the three detectors at the most critical final stage of quantification.

We have further developed combining quantitative x-ray maps from different detectors to add in the quantitative interpretation of rough samples. With two or three detectors located around the microscope, when analyzing a rough sample, the quantitative x-ray maps will look different from the three different detectors. When we combine the maps (for a specific element), we observe pixilation in areas where inaccurate data exists. If this area is neglected, then further quantitative information from these combined maps can be obtained.

Our current work involves a multi-detector energy dispersive spectrometer (EDS) and wavelength dispersive spectrometer (WDS) x-ray mapping system incorporated on a Jeol 733 microprobe (Figure 1a) and a Jeol 35CF with two EDS detectors (Figure 1b). The Jeol 733 is currently operating with four EDS x-ray detectors and three WDS detectors simultaneously. The introduction of a second EDS X-ray detector halves the time to collect a map. However, a two time statistical advantage requires four times the number of detectors and with so many detectors around the microscope, there is little room for any further additions. Consequently, a twin EDS detector in a single turret (dual detector) with only one dewar (Figure 1c) has been built and incorporated on the microscope so that we can evaluate it compared to two individual detectors.

In this talk we will be discussing the advantages and necessities of mapping using multiple EDS and WDS detectors. The talk will also discuss full-spectrum x-ray mapping and the factors that are necessary to obtain high quality results.

\section{References}

[1] D. Newbury, Microsc.Microanal 11(Suppl 2) Proceedings (2005) 1286.

[2] R. Wuhrer etal., Microsc.Microanal 1(Suppl 2) Proceedings (2005) 466.

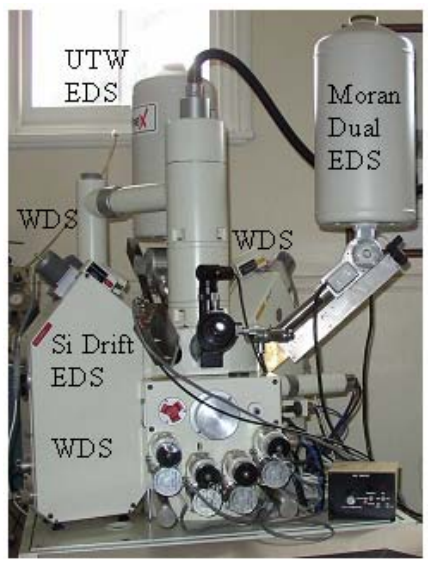

a.

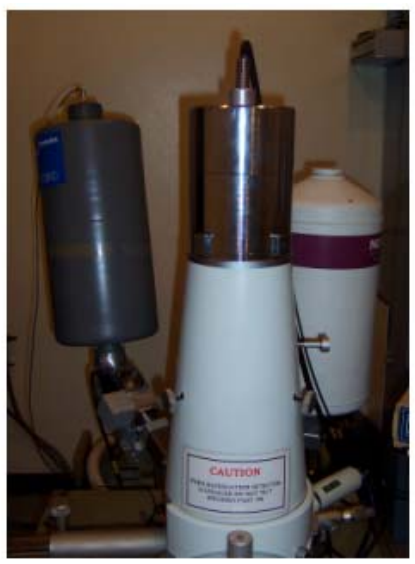

b.

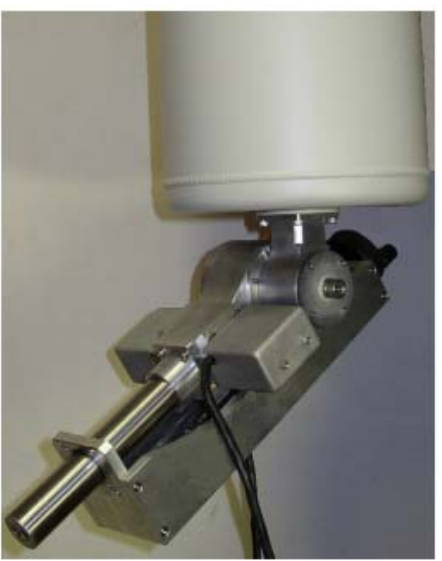

c.

Figure 1: a) JEOL 733 Microprobe with four EDS X-Ray detectors and three WDS detectors, b) JEOL 35CF with two EDS X-Ray detectors and c) A twin EDS detector in a single turret (dual detector system). 